\title{
THE FORMATION OF BRIGHTEST CLUSTER GALAXIES: THE CASE OF ABELL 407
}

\author{
LOUISE O.V. EDWARDS* \\ Department of Astronomy, Yale University \\ New Haven, CT USA 06511
}

\begin{abstract}
This article begins with a general introduction to galaxy formation and evolution and ends with a discussion of the long-term spectroscopic study: The Role of Close Companions in the Formation of Brightest Cluster Galaxies and Intracluster Light. To illustrate the power, goals and aims of this larger project, preliminary data for one of the galaxy clusters in our sample is presented, Abell 407, which appears to be caught in the act of forming its Brightest Cluster Galaxy (BCG).
\end{abstract}

\section{INTRODUCTION}

On a dark, clear night in Nova Scotia standing on your backyard porch looking up, you might spot one galaxy, our Milky Way. We live near the outer edge of one of the spiral arms of this disky-shaped collection of billions of stars. Could we could fly out of our galaxy, gazing down with a birds-eye-view, we would witness the Galaxy's spiral structure and recognize something similar to M31 (shown in the left side of the left panel of Fig 1).

The purpose of this article is to give a general review of our current understanding of the formation and evolution of galaxies and to present new data from a study currently in progress: The Role of Close Companions in the Formation of Brightest Cluster Galaxies and Intracluster Light. The introductory review goes as follows: an examination of galaxy types is found in Section - The Galaxy Zoo, a discussion of the large scale structure of the universe in Section Looking Out, and we consider the benefit of looking at galaxies during different epochs of the universe's history in Section - Looking Back. The project description includes a brief discussion of the sample, the instrumentation used, the data analysis procedure in Methods, and the presentation of our images and spectra for Abell 407 can be found in

Author to whom correspondence should be addressed: louise.edwards@yale.edu. 
Results. In the discussion we calculate very old stellar populations as well as short merging timescales for the central ellipticals. We conclude that the system will turn into a typically old large elliptical galaxy surrounded by an extended halo of intercluster light (ICL) in about 0.2 Gyrs. A full discussion of the results awaits the collection of the full sample, as argued in Future Work.
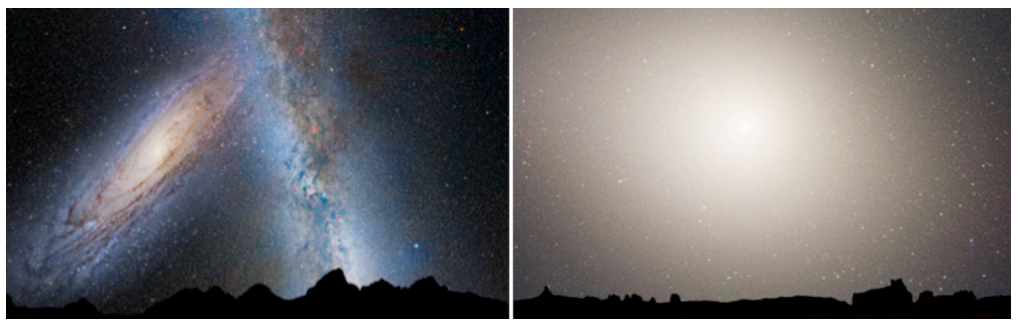

Fig 1 On the left - The predicted view of the Andromeda Galaxy (left edge) with our Milky Way (central band) from Earth in 3.75Gyrs. On the right - Predicted view 7 Gyrs after the merger. The galaxy is dominated by old red stars and has become an elliptical galaxy. (Credit: NASA; ESA; Z. Levay and R. van der Marel, STScI; T. Hallas; and A. Mellinger.)

\section{The Galaxy Zoo: Spirals, Ellipticals and Encounters}

Several of a galaxy's properties are used for developing a classification scheme. The best known of these might be Hubble's classification (Hubble 1922), the so-called 'tuning fork diagram' which sorts galaxies on the basis of their morphology, into spirals and ellipticals. Modern techniques also exploit a galaxy's size (mass or extent) as well as the analysis of the galaxy's overall colour. The latter is a proxy for discriminating between different stellar populations within a galaxy and has proved particularly important in trying to estimate the age and metal content of the system. This will become important in describing our own study, as these colours along with other diagnostics of the stellar populations can help to elucidate the evolutionary history of the galaxy.

The formation and evolution timescales can be constrained by looking at the galaxy's stellar populations, especially if single populations can be disaggregated from the whole of the galaxy. These populations are often parameterized by colour. There are many reasons that stars can be red: cooler blackbody temperatures emit more in red colours, and extinction by dust can redden starlight. In the case of tempera- 
ture, stars turn red as they age since once they finish burning their core hydrogen, they become larger and cooler. On the other hand, the small, very low mass stars start out as red, and continue on that way for billions of years. In the case of dust, short wavelength light suffers more from extinction by dust along the line of sight, so that bluer stellar populations surrounded by dust are observed as having redder colours than only their starlight would otherwise indicate. The key lies in detecting bright blue stars. These are young since the most massive stars burn hottest and are necessarily short lived. This is the population that we see associated with the actively star forming regions typical of the arms in a spiral galaxy. These massive blue stars use up their hydrogen fuel very quickly, so that they are only seen for a (cosmic) blink of an eye, a few million years. They emit most of their light at such high energies that they glow at blue colours. Additionally, the high energy photons can ionize the surrounding gas, producing a key signature - an $\mathrm{H} \alpha$ emission line at $656.3 \mathrm{~nm}$. Hence, blue colours and $\mathrm{H} \alpha$ emission lines can be used as a clock, signalling the existence of current, or at least very recent star formation.

Many grand design spirals are seen to have a nearby neighbouring galaxy, a cosmic partner in crime. Simulations show how interactions between galaxies can trigger spiral structure (Purcell et al.2011) as well as disturb the regular orbital patterns of the stars in each galaxy, forming tidal bridges and tails of stars that have been pulled off their host galaxy as the two interact via dynamical friction (Toomre and Toomre 1972). Over a few billion years the stellar orbits settle down around the combined gravitational potential of the two galaxies since they have merged into one elliptical. The product is much more red than the progenitors as the gas that existed in the two galaxies beforehand transforms in a huge burst of starformation triggered by shocks that are a result of the merger. Without any fresh gas, no new stars form and the galaxy reddens as the massive blue stars explode as supernovae and fade away. This will happen to us, as illustrated in Fig 1. The Milky Way and Andromeda are currently on a collision course, and it has been calculated that in about 5.86 billion years (van der Marel, et al.2012) the two galaxies will merge into one large elliptical, Milkomeda (Fig 1, right).

But just how ubiquitous is this phenomenon? Were the largest galaxies today formed by the same process, just through more mergers over a longer time period (Dubinski et al. 1998)? 

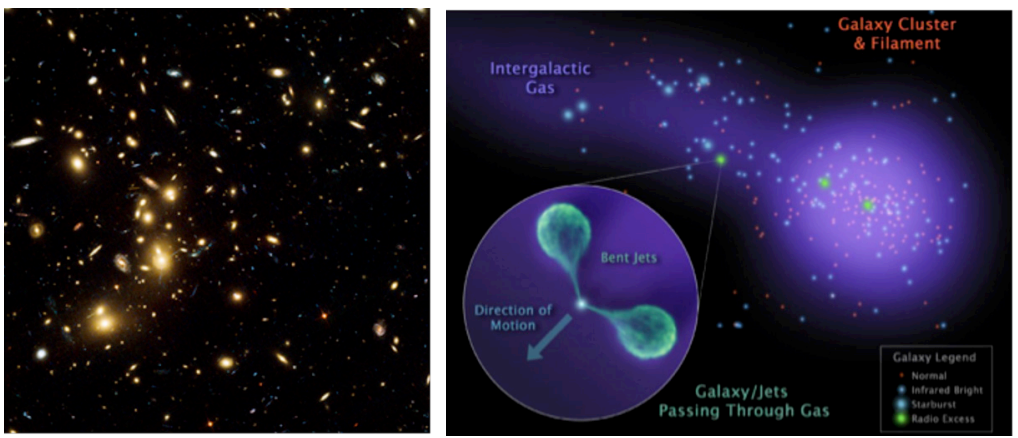

Fig 2 On the left - A Hubble Space Telescope image of Abell 2744 of the Frontier Fields. Credit: NASA, ESA, J. Lotz and M. Mountain (STScI). On the right - The galaxy cluster Abell 1763 and its cluster feeding filament coming from the direction of Abell 1770. Image credit: NASA/JPL-Caltech and L. Edwards and D. Fadda.

\section{Looking Out - A Roadmap}

The largest, brightest galaxies tend to live in particular environments. To visualize this, let's start by considering the environment of our own Milky Way. Creatively, our immediate neighbourhood is called the Local Group. This region is a collection of galaxies, including the Milky Way, Andromeda, and about 100 dwarf galaxies that have been identified as possible members (McConnachie 2012). However, most of the stellar mass in the universe is found in much larger systems. Fig 2 (left) shows the galaxies at the core of a rich cluster, wherein hundreds or thousands of galaxies are interacting gravitationally and occasionally colliding with one another as a part of their local supercluster. In turn, these superclusters are connected by filaments (Fig 2, right) where the galaxy concentration is much lower, and there are typically only $6 \times 10^{-5}$ particles in a cubic centimeter (Edwards and Fadda 2010). $6 \times 10^{-5}$ particles $/ \mathrm{cm}^{3}$ ! What is more, the regions between filaments and clusters are almost completely devoid of matter.

Our work will focus on the cores of local, rich galaxy clusters. These systems tend to have one or two extremely large galaxies at the gravitational potential of the cluster (see Fig 2, left and Fig 4). Old red stars dominate the stellar mass of the Brightest Cluster Galaxy (BCG). The galaxy morphology is elliptical, and there exists a noticeable lack of hydrogen gas clouds required to continue the generation of new stars. Thus, these galaxies have been called 'old red and dead'. However, blue stars have been found in their cores 
(Bildfell et al. 2011) as has cold molecular gas (Edge et al. 2002) and $\mathrm{H} \alpha$ emission (ex. Edwards et al. 2007, McDonald et al. 2012). BCGs are often classified as cD type, a large elliptical galaxy with an extensive stellar halo that continues out for hundreds of thousands of light-years beyond the galaxy's core. While the distribution of the starlight in many BCGs follows smooth profiles, a large fraction also contain multiple nuclei (Laine et al. 2003) and close companions (Edwards and Patton 2012).

\section{Looking Back - Our Time Machines}

How did Brightest Cluster Galaxies get so big? That is a key question which must be adressed before it can be said that we understand the origin of BCGs. Since these galaxies sit near the base of the gravitational potential well of the entire cluster, they may have experienced a higher number of mergers, gaining more stars. Alternatively, these monsters may simply have been the first to start forming, having had more time to build their stellar component. This would imply an origin independent of their special position in the galaxy cluster. Intriguingly, when we observe the cosmic microwave background (Efstathiou et al. 1992) - before there were any stars at all - some regions of the universe were slightly more dense, and some slightly less so (see top panel of Fig 3).
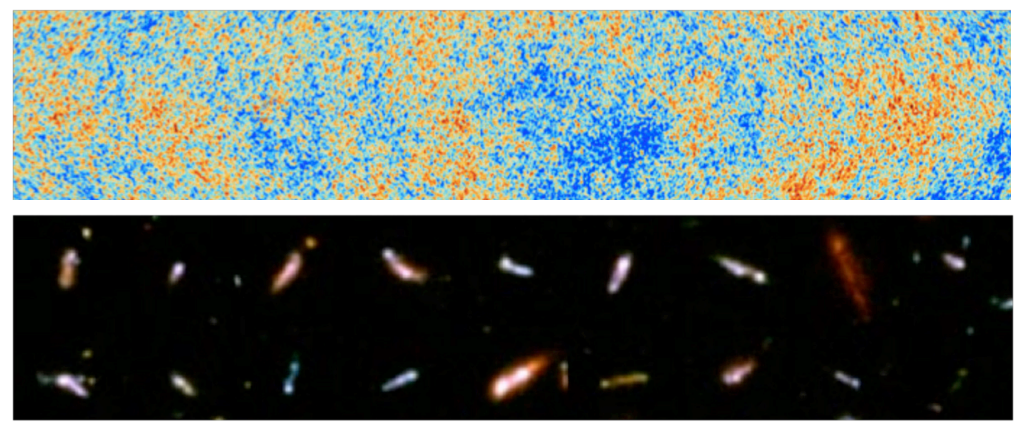

Fig 3 Top-Anisotropies in the CMB from the Planck spacecraft. Credit: ESA and the Planck Collaboration, D. Ducros. Bottom - Young, very distant massive galaxies as seen in the Hubble Ultradeep Field. These galaxies have very irregular shapes suggesting ongoing mergers. Credit: NASA, A. Straughn, S. Cohen, R. Windhorst (Arizona State University) and the UDHF team (STSci).

It is of course impossible to follow one cluster in time, as gravitational collapse and merging takes place over billions of years, but we can look at the properties of a sample of galaxies over several time 
periods. Because it takes light time to travel, observing more distant systems can track those earlier on in their evolution. In fact, before about 8 billion years ago, clusters of galaxies held only 'trainwreck' galaxies, with no clear BCG (Krick, et al . 2009). The bottom panel of Fig 3 shows galaxies from Hubble Ultra Deep Field (NASA). Many of these galaxies are seen as they were after only about two billion years from the Big Bang (Straughn et al. 2005). Large elliptical galaxies with smooth $\mathrm{cD}$ envelopes have not been observed at this early epoch. They must evolve over time.

Fast forward 8 billion years, and Fig 4 (Edwards and Patton 2012) shows how dominant and elliptical the BCGs have become in local galaxy clusters. That study showed how BCGs have a higher number of close companions compared to the other bright red galaxies of a cluster. Liu et al. (2009) find a smaller number of close pairs for BCGs that are even more local. Such results are consistent with an enhanced merger history for BCGs in the past, as well as compared to other non-BCG ellipticals. But, to further our understanding, we need to look at more direct evidence as well. Searching for tidal tails and examining the stellar populations of the intracluster light will serve as direct evidence for the origin of the diffuse stars and its possible connection to galaxy encounters.
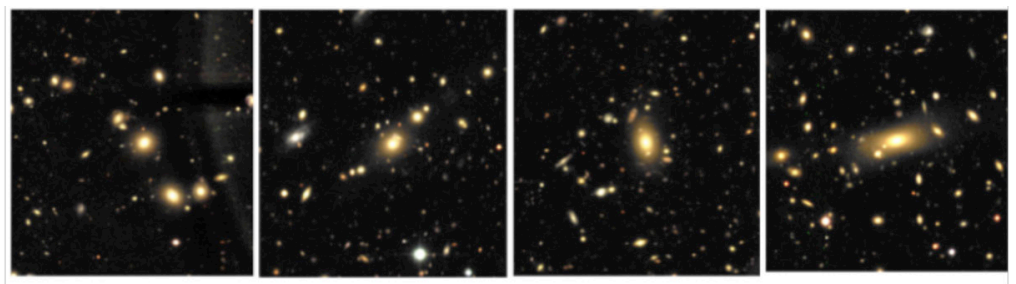

Fig 4 Images of BCGs collected by Edwards and Patton (2012) from the CFHTLS cutout service (Credit: S. Gwyn). These clusters have redshifts between $\mathrm{z}=\mathbf{0}$.20.4. By this time in cosmic history, BCGs have formed and the dominant galaxies are very red and very large. Note especially in the last three panels how the $\mathrm{cD}$ galaxy is much brighter and larger than its nearby companions.

\section{METHODS}

Our study will measure relative ages, metal content and star formation rates in a selection of BCGs, their nearby neighbours, and the surrounding intracluster light (ICL). We start with the NOAO Fundamental Plane survey's sample of 100 nearby rich clusters (Smith 
et al. 2004). Choosing clusters that are observable from the WIYN telescope in Arizona, and at redshifts commensurate with angular source sizes fitting into the instrument field of view, 24 galaxies are chosen for observation. Over three observational campaigns, we have already been able to observe 12 of these systems using integral spectroscopy. Integral field units (IFU) are instruments that recover an entire spectrum for each small 'pixel' on the sky. Illustrated in Fig 5 , IFUs allow us in one go, to get the light signatures from all three of the cluster core components. This section presents the instrument background and observations and the data reduction. The results and discussion of one of our clusters, Abell 407, follow.

\section{Instrument Background and Observations}

The data was collected using the SparsePak instrument (Bershady et al. 2004), which is mounted on the WIYN $3.5 \mathrm{~m}$ telescope on Kitt Peak in Arizona. The spectrograph has 82 fibers arranged in a twodimensional array, with a central core of 75 fibres and 7 sky fibres on the periphery (see Fig 5). Each fibre captures light from a circular field of 4.7" in diameter. The central core has on-sky dimensions of 72 " $x$ 71.3", while the sky fibres are another 25 " away from the core. At the distance of Abell 407, this translates to 19.7 by 19.4 thousand lightyears, covering both the BCG and its close companions. A superposition of three pointings of the SparsePak grid fills the spaces between the fibres so that an integral image can be constructed at any wavelength between $400 \mathrm{~nm}$ and $700 \mathrm{~nm}$.

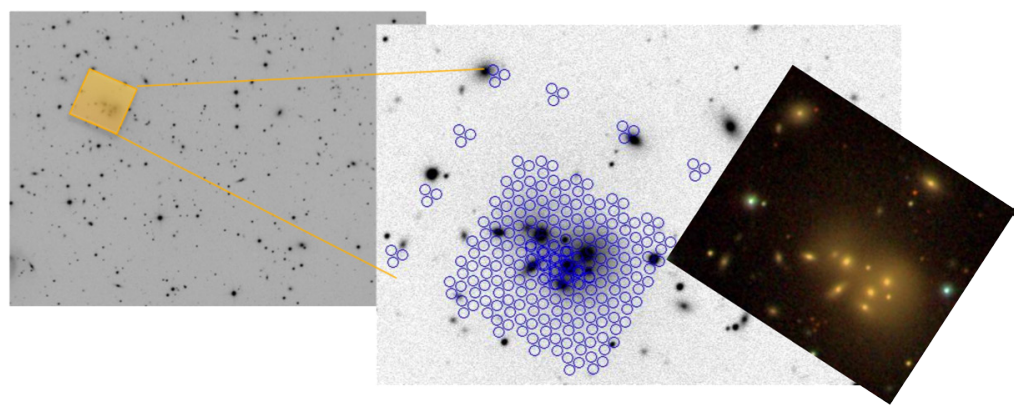

Fig 5 Left - Wide field r'-band image from the Sloan Digital Sky Survey (SDSS), with overlay of Sparcepak instrument field of view in middle panel. Right -3 colour SDSS image of the core of Abell 407. The dimensions of cut out are 72" x 71.3" or 19.7 by 19.4 thousand lightyears at the distance of Abell 407 . 
Throughout the observing run, the instrument had a gain of 0.219 e-/adu and a readout noise of 3.4. For our spectrograph configuration, we used the grating600@10.1 with a central wavelength of 570nm and the GG-420 filter to cut second order spectral lines.

Before observing the BCGs each night, sets of dome flats, arcs, biases and dark frames were taken. Flats are exposures taken on the dome to show defects of the CCD or other lighting problems; arcs are taken for wavelength calibration purposes; biases are zero second exposures that show the noise of the camera; and darks are taken with no light on the camera to reveal problems like hot pixels, or pixels with high leakage. Along with a standard star for flux calibration, all data for Abell 407 was taken in good weather conditions over the span of four nights between Nov 25 and 30, 2013. Data for 11 other clusters, from two other runs in 2012-2013 will be discussed elsewhere, along with the observations of additional clusters from Fall 2014.

\section{Data Reduction}

The data are pre-processed in the normal way using IRAF (Tody 1993). We subtracted detector bias and dark, and divided the normalized flat-field response of the CCD. We removed cosmic rays using the IRAF task lacosic (van Dokkum 2001) and subtracted the sky level using non-polluted Sparsepak sky fibres. We used the values from the NASA Extragalactic Database ${ }^{1}$ to remove Galactic extinction and to correct for the published galaxy cluster redshift. Finally, we applied the flux calibration using the standard star.

\section{RESULTS}

Fig 5 shows an SDSS image of the core of the rich galaxy cluster, Abell 407. This is a very rare object as it has a great many large galaxies at the center of a poor cluster, it has even been referred to as the 'most nightmarish' of multiple nuclei central galaxies by Schneider and Gunn (1982). Some extended diffuse radio emission has been detected (Liuzzo et al. 2010), but the cluster is not a strong X-ray source. The continuum graph of Fig 6 displays the total intensity in the range of 5,500 to 6,000 angstroms for each fibre. This reconstructed image clearly shows light at the positions of the BCG (object N), and

1 NASA Extragalactic Database is hosted by this site http://ned.ipac.caltech.edu/. 

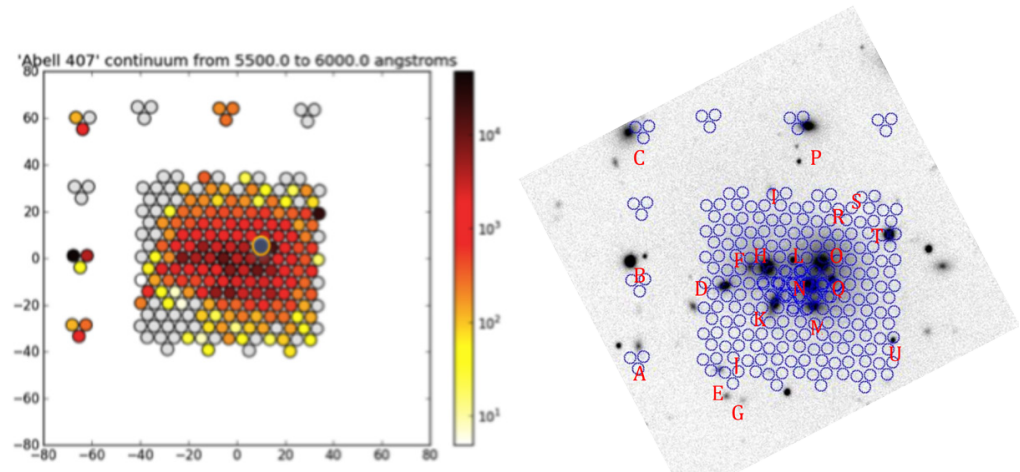

Fig 6 Left - reconstructed image of the red end of the spectrum for all galaxies and starlight in the Sparsepak field of view. Credit: Hannah Alpert and Tara Abraham, undergraduate students at Yale University. Right - An SDSS r'band image with three pointings of the Sparsepak fibres is overlaid on top. Sources A-U have been labeled in accordance to Table 1. Credit: Figure makes use of Jenny Power's Sparsepak visualization program (NOAO). The image scale uses false colours.

other elliptical galaxies and foreground stars. The grey fibres represent outlier data with a signal-to-noise ratio too low to be meaningful.

Seven bright and 2 faint nuclei occupy the core of the cluster, within 10 kly of each other. An examination of Fig 6 reveals that all are within the projected area of the diffuse stellar halo - the false red and orange colours show the stellar envelope extends not quite to the outer edge of the IFU. In this section, we present the colours and magnitudes of the main galaxies as labeled in Fig 6 (right) as well as their spectra and velocities.

\section{Colours and Magnitudes}

Most of the highest luminosity objects are found to have very red colours, with the reddest colours being in the outer envelope of the brightest object (our O) and becoming slightly redder with increasing radius (Mackie 1992). By contrast, Schneider and Gunn (1982) found that the galaxies were slightly redder than the ICL.

Within the footprint of our WIYN data, we searched the Sloan Digital Sky Survey (SDSS) and found 21 photometric sources with r' magnitudes brighter than 21.9. Of these, Table 1 shows that seven are identified as stars by the SDSS star/galaxy separator, but only three $(\mathrm{B}, \mathrm{U}$ and $\mathrm{T})$ are clearly bright stars based on our spectra and 


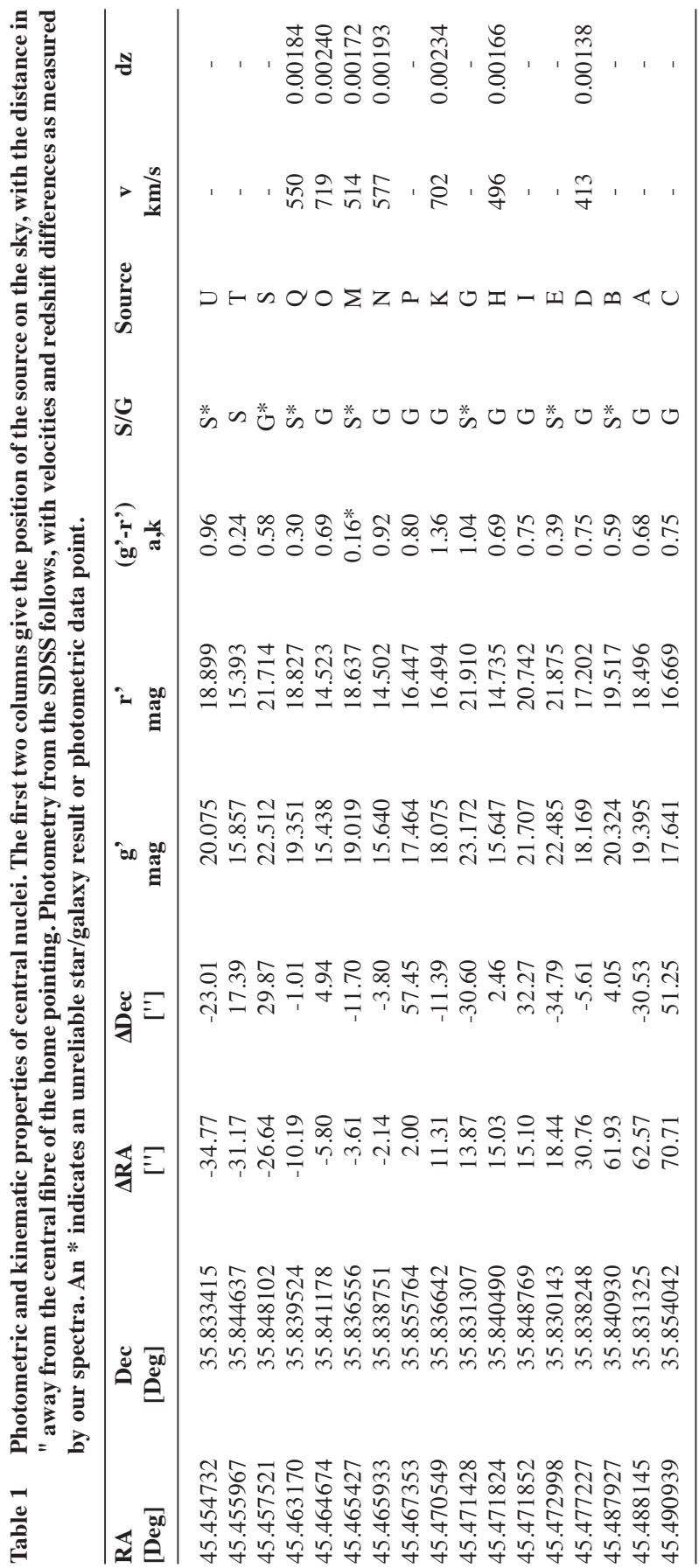


further inspection of the SDSS images. The table also shows that all galaxies are quite red, with (g'-r') colours greater than 0.35. Notable exceptions are sources $\mathrm{T}$ and $\mathrm{Q}$, which are classified as stars by the SDSS. T falls squarely into one of the fibres, and the source spectrum is very clearly that of a foreground star. Source Q is less clear in that only part of its light falls into one of our fibres. Q's light is heavily polluted by that from $\mathrm{O}, \mathrm{N}, \mathrm{M}$, and presumably the ICL. M, is clearly a galaxy by its spectrum and light profile. Its SDSS photometry is flagged as unreliable.

The brightest 3 galaxies $(\mathrm{O}, \mathrm{N}, \mathrm{H})$ are very close in r'-mag, separated only by $0.2 \mathrm{mag}$, which is roughly the error. This means there is no clear optical BCG. K-band photometry in the near-IR can be a better measure of stellar mass, but only $\mathrm{O}$ and $\mathrm{C}$ and $\mathrm{P}$ have published photometry from the Two Micron All-Sky Survey (2MASS). From the Infrared Science Archive, the 2MASS K-band magnitudes are 10.409, 13.726 and 13.202, respectively. True $\mathrm{cD}$ galaxies are often the $1 \mathrm{st}$ ranked galaxy by a magnitude or more (ex. Abell 2029) - underscoring that Abell 407 has no dominant galaxy, therefore has BM Type II (Corwin 1974). When comparing the intensity at the red end of the spectrum to the blue end and (g'-r') colours of the ICL, H, N and O, we find that $\mathrm{N}$ has the reddest colours, with $\mathrm{H}, \mathrm{O}$ and the ICL being indistinguishable in redness.

\section{Spectra and velocities}

By looking at the source velocity along the line of sight, we identified two foreground stars. None of the bright sources are identified as interloping galaxies.

Fig 7 shows the bright spectra we combined from all central sources within our field of view. The deep absorption lines of $\mathrm{NaD}, \mathrm{Mgb}$ and $\mathrm{H} \beta$ are centered at nearly the same wavelength, consistent with all of the galaxies being physical members of the cluster. This is consistent with the data put forward by the NOAO Fundamental plane survey that these galaxies form a distinct subcluster. There are two notable exceptions: 1) Star T is clearly in the foreground and when the redshift applied is removed, the hydrogen absorption lines are clear. 2) Source R is too faint and too low $\mathrm{S} / \mathrm{N}$ to resolve any absorption lines. 


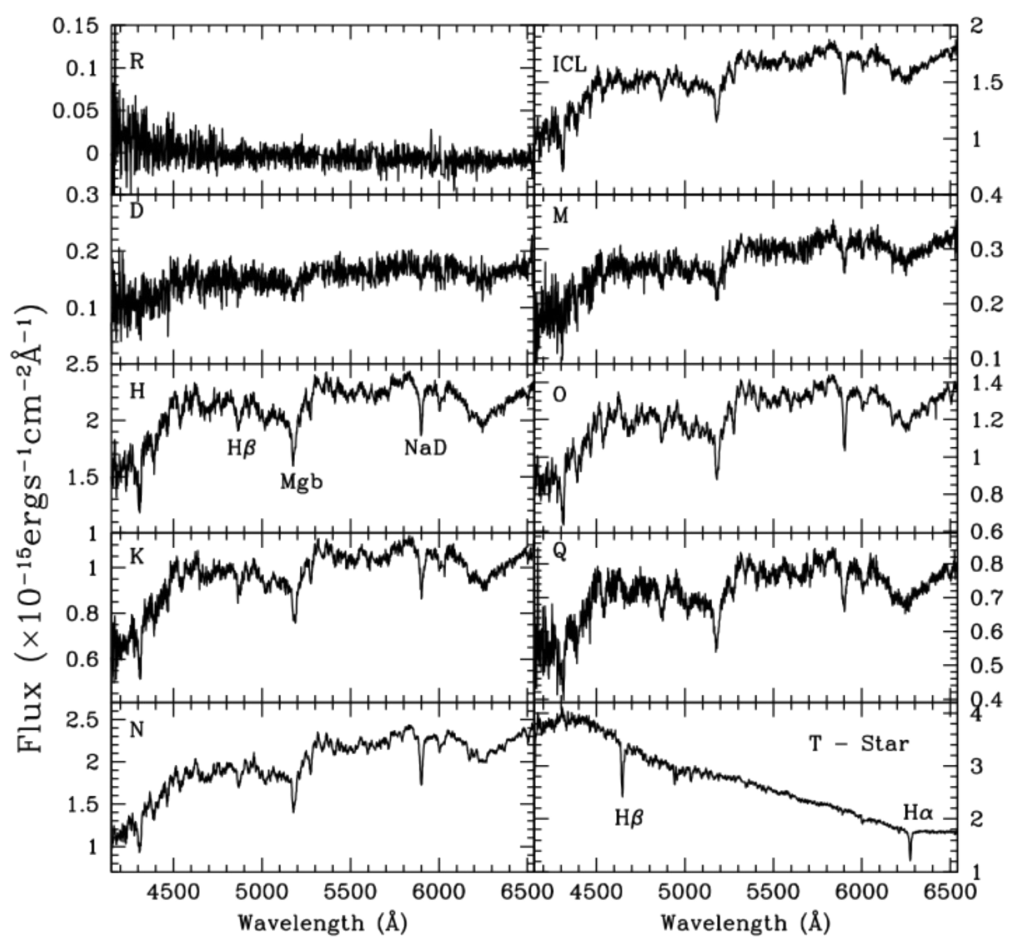

Fig 7 Combined spectra of the brightest sources within the Sparsepak field of view. The sources are labeled as defined in Fig 6. Bright absorption lines are labeled in panel $\mathbf{H}$. All spectra have been deredshifted to the published cluster redshift of 0.0462 .

\section{DISCUSSION}

Mackie (1992) conclude that the red envelopes they found in Abell 407 cluster galaxies could be from enhanced metallicity at the outer edges, from asymptotic giant branch stars (resulting from recent mergers) or from dust. It could also be that inner parts are bluer from current starformation, but we find no evidence for any strong optical emission lines, suggesting there is no significant ongoing star formation or active galactic nucleus (AGN) activity in the core. Our colours and spectra for all the central galaxies are consistent with a dominant old population.

To quantify this, we measure line strengths (Vazdekis 2011) on all of the bright sources of Table 1 to measure the Lick indices (depth of absorption lines, Worthey 1994). Most importantly, the results give 

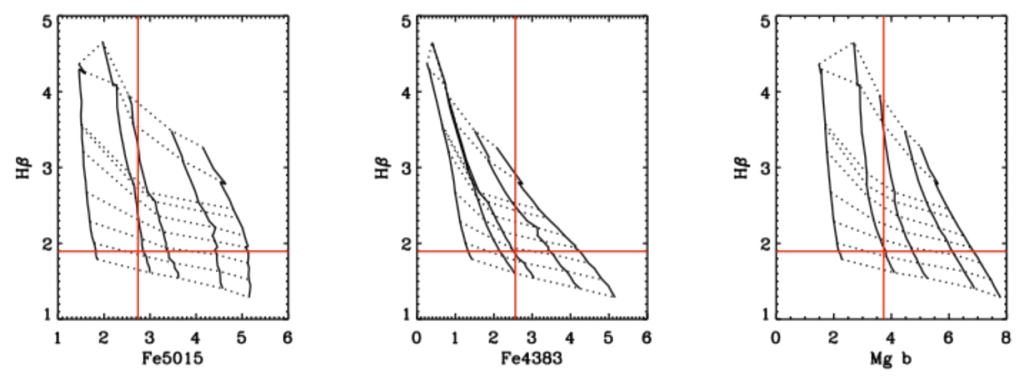

Fig 8 Results from running the population synthesis code, EZ_Ages. Dotted lines are of constant age, increasing downward. The dotted line at the top represents an age of $1.2 \mathrm{Gyr}$, followed by $1.5,2.5,2.8,3.5,5.0,7.0,10.0$ and 14.1 Gyrs. Solid lines are of constant metallicity, increasing to the left, starting with $[\mathrm{Fe} / \mathrm{H}]=-1.3$ and continuing on to $-0.7,-0.4,0.0$ (solar), and +0.2 . The red cross indicates the best-fit point for the ICL spectral indices. The stars are old and metal poor.

the relative strength of $\mathrm{H} \beta$ and Mgb absorption. Although there is some degeneracy in these population indicators (Carter et al. 2009), in general for old populations, as the $\mathrm{H} \beta$ decreases, the age of the system increases, and as Mgb increases, the metallicity does as well.

Using the EZ_Ages population synthesis code(Greves and Schiavon 2008), we translate the indices into ages and metallicites based on the best-fit to stellar population models. The results are shown in Fig 8 for the integrated spectrum that belongs to the ICL. We compute an age of $9.04 \mathrm{Gyr}$ and a metallicity of $[\mathrm{Fe} / \mathrm{H}]$ of -0.71 ; these are old, metal poor stars. For the individual bright galaxies, the $\mathrm{H} \beta$ indices are even lower, implying even older stellar populations.

Schneider and Gunn (1982) performed a spectroscopic study on the galaxies of this cluster, collecting excellent spectra of the central nuclei, but not being able to achieve a high $\mathrm{S} / \mathrm{N}$ spectrum of the ICL. These authors find that the three largest galaxies have velocity dispersions within $200 \mathrm{~km} / \mathrm{s}$, which they related to a merging time of about a billion years. This would then be a very rare system, as we are catching the poor cluster, not in a steady state of inflow, but at a special time in history before all of the galaxies have converged into one cD galaxy. However, as these objects are in fact quite scarce in the local universe, this is possible. We compute the dynamical friction timescale of our measurements of the large galaxies. By using a velocity of $250 \mathrm{~km} / \mathrm{s}$ and a companion mass of $6.63 \times 10^{11}$ solar masses, and a distance of $25 \mathrm{kly}$, we estimate the system will have merged into one large cD galaxy in only 0.2 Gyr. We concur with Mackie 
(1992) who claim that Abell 407 is in the process of building a larger cD galaxy. We detect ICL that echoes the major nuclei of the center in its morphology, and as the ICL does not exist much past the main nuclei, which is similar to the findings of Feldmeier et al. (2004), who find the little ICL that exists, closely follows the morphology of the largest galaxies of BM type II-III clusters.

\section{CONCLUSION}

We have shown that the central region of Abell 407 is home to 7 bright galaxies, where the 1st, 2nd and 3rd ranked members are all equal within the photometric errors. We confirm there is no clear cD galaxy, and that the system is BM type II. We have measured red colours for all three as well as absorption line strengths. The colours and absorption line strengths suggest a dominant old stellar population for all the bright galaxies, with an age of $9.044 \mathrm{Gyr}$ with a low sub-solar metallicity for the ICL. We found no emission lines in the central, consistent with very old stellar populations in all nuclei, as well as in the ICL. Based on the paucity and irregular morphology of the ICL, we conclude that the brightest red galaxies are currently interacting, building a cD galaxy and as well as an extended ICL.

Using the redshifts of the individual galaxies compared to the cluster as a whole, we estimate the merger timescale by calculating the dynamical friction timescale, which turns out to be less than 0.2 Gyr. This is consistent with Abell 407 currently building a cD galaxy and ICL. Dubinski et al. (1998) predicts that morphologically relaxed clusters should have more ICL. In this unrelaxed cluster, we see no strong sign of ICL past $10 \mathrm{kly}$ from the core. Additionally, the ICL morphology echoes the morphology of the large galaxies at the center of the cluster, and the colours are equally as red as the main galaxies.

\section{Future Work}

Once we have finished collecting our data, we will determine ages, metallicites, and star formation rates for a well determined sample of 15-20 nearby cluster cores. In addition to similar analysis on each of the clusters individually, we will be able to make a statement about the nature of local clusters in general and any links between the ICL, BCG and close companions. Does the ICL increase constantly with $\mathrm{BM}$ type or metallicity of the stellar populations? At what point in 
the evolution do the blue cores of BCGs come into existence, and do they correlate with other cluster properties we have not discussed here, like AGN activity of X-ray brightness?

\section{Final Remarks}

We began this article with a general overview of galaxy formation and evolution. To end, let us consider the scale of Abell 407. It has a characteristic radius of roughly $20 \mathrm{kly}$ and is about 708 million light years from the Sun. We suggest that from the time we saw the galaxies as they were, it would take $\sim 0.2$ Gyr for them to merge. Thus, 'today' this cluster would look quite different. It would not have 7 bright nuclei surrounded by a lumpy ICL, but rather would be dominated by one extremely large $\mathrm{cD}$ type galaxy, with old stars having been flung further out during the galaxy encounters, increasing the size of the ICL. Perhaps, during these huge mergers, small amounts of new stars would have been born, or would have triggered an AGN outburst, and the spectra would show $\mathrm{H} \beta$ in emission, rather than absorption. We would have to survive nearly another billion years to know about Abell 407's future for certain. But, amazingly, by looking at samples of galaxy clusters throughout several epochs, we will be able to quantify the plausibility of that scenario.

Acknowledgements This project made use of several online public astronomical databases. This includes: NASA/STSci and Hubble Space telescope images; NASA/IPAC Extragalactic Database (NED) which is operated by the Jet Propulsion Laboratory, California Institute of Technology, under contract with the National Aeronautics and Space Administration; the NASA/ IPAC Infrared Science Archive, which is operated by the Jet Propulsion Laboratory, California Institute of Technology, under contract with the National Aeronautics and Space Administration; the SDSS where funding for SDSS-III has been provided by the Alfred P. Sloan Foundation, the Participating Institutions, the National Science Foundation, and the U.S. Department of Energy Office of Science. The SDSS-III web site is http://www.sdss3.org/. SDSS-III is managed by the Astrophysical Research Consortium for the Participating Institutions of the SDSS-III Collaboration including the University of Arizona, the Brazilian Participation Group, Brookhaven National Laboratory, Carnegie Mellon University, University of Florida, the French Participation Group, the German Participation Group, Harvard University, the Instituto de Astrofisica de Canarias, 
the Michigan State/Notre Dame/JINA Participation Group, Johns Hopkins University, Lawrence Berkeley National Laboratory, Max Planck Institute for Astrophysics, Max Planck Institute for Extraterrestrial Physics, New Mexico State University, New York University, Ohio State University, Pennsylvania State University, University of Portsmouth, Princeton University, the Spanish Participation Group, University of Tokyo, University of Utah, Vanderbilt University, University of Virginia, University of Washington, and Yale University; the CFHTLS which is based on observations obtained with MegaPrime/ MegaCam, a joint project of CFHT and CEA/IRFU, at the CanadaFrance-Hawaii Telescope (CFHT) which is operated by the National Research Council (NRC) of Canada, the Institut National des Science de l'Univers of the Centre National de la Recherche Scientifique (CNRS) of France, and the University of Hawaii. This work is based in part on data products produced at Terapix available at the Canadian Astronomy Data Centre as part of the Canada-France-Hawaii Telescope Legacy Survey, a collaborative project of NRC and CNRS. The IFU data for Abell 407 was taken at the WIYN Observatory which is a joint facility of the University of Wisconsin-Madison, Indiana University, Yale University, and the National Optical Astronomy Observatory.

The National Optical Astronomy Observatory (NOAO) consists of Kitt Peak National Observatory near Tucson, Arizona, Cerro Tololo Inter-American Observatory near La Serena, Chile, and the NOAO Gemini Science Center. NOAO is operated by the Association of Universities for Research in Astronomy (AURA) under a cooperative agreement with the National Science Foundation.

Great thanks and appreciation also goes out to Sean McGee who helped with the project proposal as well as Hannah Alpert, Tara Abraham and Saisneha Koppaka who helped with gathering, reducing and visualizing the Abell 407 data. Finally, I would like to thank the NSIS for inviting me to Halifax in May 2014 for a very enjoyable evening.

\section{REFERENCES}

Bershady, M.A., Andersen, Harker, J., et al . (2004). SparsePak: A Formatted Fiber Field-Unit for The WIYN Telescope Bench Spectrograph. I. Design, Construction, and Calibration. PASP. 116:565-590.

Bildfell, C., Hoekstra, H., Babul, A., et al. (2008). Resurrecting the red from the dead: optical properties of BCGs in X-ray luminous clusters. MNRAS. 389:1637-1654. 
Carter, D. Smith, D.B., Percival, S.M., et al. (2009). Optical and nearinfrared colours as a discriminant of the age and metallicity of stellar populations. MNRAS. 397:695-708.

Corwin, H.G. (1974). Abell galaxy cluster magnitudes and the Hubble diagram. AJ. 79:1356-1364.

Dubinski, J. (1998). The Origin of the Brightest Cluster Galaxies. ApJ. 502:141-149.

Edge, A.C., Wilman, R.J., Johnstone, R.M., et al. (2002). A survey of molecular hydrogen in the central galaxies of cooling flows. MNRAS. 227:49-62.

Edwards,L.O.V.,Patton,D.R.(2012). Close companions to brightest cluster galaxies: spport for minor mergers and downsizing. MNRAS. 425:287-295.

Edwards, L.O.V., Fadda, D. (2010). The First Bent Double Lobe Radio Source in a Known Cluster Filament: Constraints on the Intrafilament Medium. ApJL. 724:143-147.

Efstathiou, G.; Bond, J.R.; White, S.D.M. (1992). COBE background radiation anisotropies and large-scale structure in the universe. MNRAS. 258:1-6.

Feldmeier, J.J., Mihos, C.J., Morrison, H.L., et al. (2004). Deep CCD Surface Photometry of Galaxy Clusters. II. Searching for Intracluster Starlight in Non-cD clusters. ApJ. 609:617-637.

Graves, G., Schiavon, R. (2008). Measuring Ages and Elemental Abundances from Unresolved Stellar Populations: Fe, Mg, C, N, and Ca. ApJS. 177:446-464.

Hubble, E.P. (1922). A general study of diffuse galactic nebulae. ApJ. 56:162-199.

Krick, J.E., Surace, J.A., Thompson, D. et al. (2009). Galaxy Clusters in the IRAC Dark Field. II. Mid-Infrared Sources. ApJ. 700:123-136.

Laine, S., van der Marel, R.P., Lauer, T.R. (2003). Hubble Space Telescope Imaging of Brightest Cluster Galaxies. AJ. 125:487-505.

Liu F.S., Mao S., Deng Z.G., Xia X.Y., Wen Z.L. (2009). Major dry mergers in early-type brightest cluster galaxies. MNRAS, 396:2003-2010.

Liuzzo, E.; Giovannini, G.; Giroletti, M.; Taylor, G.B . (2010). Parsecscale properties of brightest cluster galaxies. $A \& A$. 516:1-16.

Mackie, G. (1992). The stellar content of central dominant galaxies. II Colors of cD envelopes. ApJ. 400:65-63.

McConnachie, A. (2012). The observed properties of the Dwarf Galaxies in and around the Local Group. AJ. 144:4-40.

McDonald, M., Veilleux, S., Rupke, D.S.N. (2012). Optical Spectroscopy of $\mathrm{H} \alpha$ Filaments in Cool Core Clusters: Kinematics, Reddening, and Sources of Ionization. ApJ. 746:153-176.

Purcell, C.W., Bullock, J.S., Tollerud, E.J., et al. (2011). The Sagittarius impact as an architect of spirality and outer rings in the Milky Way. Nature. 477:301-303.

Smith, R.J., Hudson, M.J., Nelan, J.E., et al . (2004). NOAO Fundamental Plane Survey. I. Survey Design, Redshifts, and Velocity Dispersion Data. AJ. 128:1558-1569. 
Straughn, A.N., Cohen, S.H., Ryan, R.E., et al. (2005). Tracing Galaxy Assembly: Tadpole Galaxies in the Hubble Ultra Deep Field. ApJ. 639:724-730.

Tody, D. (1993). IRAF in the Nineties in Astronomical Data Analysis Software and Systems II, A.S.P. Conference Ser., Vol 52, eds. R.J. Hanisch, R.J.V. Brissenden, and J. Barnes, 173.

Toomre, A., Toomre, J. (1972). Galactic Bridges and Tails. ApJ, 178: 623-666.

Schneider, D. P., Gunn, J. E. (1982). V ZW 311 - The once and future cD. ApJ. 263:14-22.

van der Marel, R.P., Besla, G., Cox, T.J. (2012). The M31 Velocity Vector. III. Future Milky Way M31-M33 Orbital Evolution, Merging, and Fate of the Sun. ApJ. 753:9-30.

van Dokkum, P.G. (2001). Cosmic-Ray Rejection by Laplacian Edge Detection. PASP. 113:1420-1427.

Vazdekis, A. (2011). LECTOR: Line-strengths in One-dimensional ASCII Spectra. Astroph Source Code Library record ascl:1104.006.

Worthey, G. (1994). Comprehensive stellar population models and the disentanglement of age and metallicity effects. ApJS. 95:107-149. 